\title{
Small interfering RNA silencing of interleukin-6 in mesenchymal stromal cells inhibits multiple myeloma cell growth
}

\begin{abstract}
Studies demonstrated that mesenchymal stromal cells (MSC) from bone marrow stroma produced high concentration of interleukin-6 (IL-6) that promoted multiple myeloma cell growth. In view of the failure of IL-6 monoclonal antibody therapy to demonstrate substantial clinical responses in early clinical trials, more effective methods are needed in order to disrupt the favourable microenvironment provided by the bone marrow stroma. In this study, we evaluated the short interfering RNA (siRNA)-mediated silencing of IL-6 in MSC and the efficacy of these genetically modified MSC, with IL-6 suppression, on inhibition of U266 multiple myeloma cell growth. IL-6 mRNA and protein were significantly suppressed by $72 \mathrm{~h}$ post IL-6 siRNA transfection without affecting the biological properties of MSC. Here we show significant inhibition of cell growth and IL-6 production in U266 cells co-cultured with MSC transfected with IL-6 siRNA when compared to U266 cells co-cultured with control MSC. We also show that the tumour volume and mitotic index of tumours in nude mice coinjected with U266 and MSC transfected with IL-6 siRNA were significantly reduced compared to tumours of mice co-injected with control MSC. Our results suggest potential use of RNA interference mediated therapy for multiple myeloma.
\end{abstract}

Keyword: Mesenchymal stromal cells; Interleukin-6; siRNA; RNA interference; Multiple myeloma 